\title{
Prescribing patterns of antihypertensive drugs in tertiary care teaching hospital
}

\author{
Rajesh Kumar Suman $^{1 *}$, H. K. Singh ${ }^{1}$, Vithal G. Patil ${ }^{2}$
}

${ }^{1}$ Department of Pharmacology, Hind Institute of Medical Sciences, Mau, Ataria, Sitapur, Uttar Pradesh, India

${ }^{2}$ Bharti Vidyapeeth Dental College, Kharghar, Navi Mumbai, Maharashtra, India

Received: 10 February 2021

Revised: 10 March 2021

Accepted: 11 March 2021

\section{*Correspondence:}

Dr. Rajesh Kumar Suman,

Email: rajeshsuman2043@mail.com

Copyright: ( $)$ the author(s), publisher and licensee Medip Academy. This is an open-access article distributed under the terms of the Creative Commons Attribution Non-Commercial License, which permits unrestricted non-commercial use, distribution, and reproduction in any medium, provided the original work is properly cited.

\begin{abstract}
Background: Prescribing patterns provides prescribing behaviors of prescriber. Hypertension is most common cardiac complication among middle and old age population. Thus, study about prescribing trends helps to select appropriate drug for treatment of hypertension. The study aim was to analyze the patterns of antihypertensive drug prescribed in patients diagnosed with hypertension.

Methods: The study was cross sectional and observational study. A questionnaire was specifically designed factoring patients' demographical profile, diagnosis of disease, drug regimen.

Results: The totals of 100 patients were analyzed for the prescribing patterns of antihypertensive drug maximum patients belonged to the age group of 61-80. The proportion of male (62\%) patients was more as compared to female patients (38\%). Total drug prescribed was 246 in 100 prescriptions. Average drug per prescription was 2.46. Among 246 drugs, $97 \%$ were antihypertensive prescribed and $3 \%$ were other concomitant drugs. $65 \%$ single drug prescribed, $25 \%$ two drug prescribed and $10 \%$ three drug prescribed. Most commonly single antihypertensive drug prescribed was telmisartan. Most commonly two antihypertensive drug prescribed was telmisartan + hydrochlorothiazide. Most commonly three antihypertensive drug prescribed was telmisartan + amlodipine + hydrochlorothiazide. Among total 246 drug prescribed, angiotensin receptor blocker was prescribed (ARB) 28\%, calcium channel blocker (CCB) $17 \%$, angiotensin converting enzyme inhibitor (ACEI) $12 \%$, beta blocker 5\% followed by ARB + diuretics $13 \%$, ACEI + diuretics $8 \%$, beta blocker + $\mathrm{CCB} 4 \%, \mathrm{ARB}+\mathrm{CCB}+$ diuretics 7\%, ARB + beta blocker + diuretics 3\% and 3\% other drug prescribed. There was no fixed dose combination prescribed and no injectable prescribed in present study.

Conclusions: The most common drug prescribed was ARB as single drug therapy. The most common drug which was used for combination therapy was diuretics.
\end{abstract}

Keywords: Prescribing patterns, Diuretics, Hypertension

\section{INTRODUCTION}

Hypertension has been reported to be the strongest modifiable global risk factor for cardiovascular morbidity, mortality as well as health burdens. The world has consistently identified an important and independent link between hypertension and various disorders, especially coronary heart disease, stroke, congestive heart failure and impaired renal function as per Some Epidemiological studies. ${ }^{1}$

The leading risk resulting in considerable death and disability worldwide is hypertension and accounted for 9.4 million deaths and $7 \%$ of disability adjusted life years in 2010. In India, the situation is more alarming as hypertension attributes for nearly $10 \%$ of all deaths. Prevalence of hypertension in India is reported to vary 
from $4-15 \%$ in urban and $2-8 \%$ in rural population. It is estimated that the worldwide prevalence of hypertension would increase from $26.4 \%$ in 2000 to $29.2 \%$ in 2025 . Epidemiological studies also demonstrate that prevalence of hypertension is increasing rapidly among Indian urban and rural populations. ${ }^{2,3}$

The various reasons for hypertension are socio-economic, behavioural, sedentary life style, Nutritional and poor health maintenance, the poor controlling of hypertension leads to further Progression cardiovascular complications like ischemic heart disease, heart failure, stroke and chronic renal insufficiency. ${ }^{4,5}$

Currently, primary care physicians can choose from numerous pharmacological agents to treat hypertension. Various classes of drug available to treat hypertensive patient. The choice of drug depends upon patient and other related factors. Recommendations of a variety of guidelines are available for treatment of hypertension, such as WHO, ISH guidelines and recently the recommendations given by Joint National Committee (JNC-VIII) of USA on prevention, detection, evaluation and treatment of high blood pressure which suggests the rationale administration of drugs by providing algorithms for the treatment as per the stages of hypertension. ${ }^{6,7}$ The prescribing trend for hypertension is changing day by day as recent advances coming across to enhance the treatment guidelines for hypertension patients. The present study has been desgined to evaluate current prescribing trend in our teaching hospital for the hypertension patients.

\section{Objectives}

Objectives were to analyze the patterns of antihypertensive drug prescribed in medicine OPD patients.

\section{METHODS}

\section{Study design}

Prospective-open labeled, cross sectional and observational survey

\section{Sample size}

Total 100 patients were recruited for the study

\section{Study place and duration}

The study is conducted in MGM Medical College between July 2016 to Jan 2017.

\section{Patient selection}

\section{Inclusion criteria}

Patient attending Medicine OPD and giving consent to participate in the study.

\section{Exclusion criteria}

Patient who were seriously sick (emergency) and IPD patients.

\section{Study material}

A specially designed data entry format was used to record patients' details like patient name, age, sex, drug regimen, drug dose.

The data has been put in excel sheet, analysed and results is presented in percentage.

\section{RESULTS}

The totals of 100 patients were analyzed for the prescribing patterns of antihypertensive drug in the medicine department. Maximum patients belonged to the age group of 61-80 years (42\%) followed by age group of 41-60 years $(38 \%)$. The proportion of male $(62 \%)$ patients was more as compared to female patients $(38 \%)$ (Table 1).

Total 100 prescriptions of patients who were visited our medicine OPD for treatment of hypertension was analyzed. Total drug prescribed was 246 in 100 prescriptions. Average drug per prescription was 2.46. Among 246 drugs, $97 \%$ were antihypertensive prescribed and $3 \%$ were other concomitant drugs prescribed like aspirin, atorvastatin, vitamin B complex, thyroxine, ranitidine, pantaprazole, metformin, glimiperide etc. Most commonly single antihypertensive drug prescribed was telmisartan. Most commonly two antihypertensive drug prescribed was telmisartan + hydrochlorothiazide. Most commonly three antihypertensive drug prescribed was telmisartan + amlodipine + hydrochlorothiazide (Table 1).

Table 1: Pattern of demographic data of medicine OPD patients.

\begin{tabular}{|lll|}
\hline Sr. no. & Parameters & Results \\
\hline $\mathbf{1}$ & Total no. of sample & 100 \\
\hline $\mathbf{2}$ & OPD sample & 100 \\
\hline $\mathbf{3}$ & Age ( year) & $\%$ of patients \\
\hline & $20-40$ & 20 \\
\cline { 2 - 3 } & $41-60$ & 38 \\
\hline $\mathbf{4}$ & Gender & 42 \\
\hline
\end{tabular}




\begin{tabular}{|lll|}
\hline Sr. no. & Parameters & Results \\
\hline & Male & 62 \\
\cline { 2 - 3 } & Female & 38 \\
\hline $\mathbf{5}$ & Total drug prescribed & 246 \\
\hline $\mathbf{6}$ & Average drugs/prescription & 2.46 \\
\hline $\mathbf{9}$ & Most commonly single antihypertensive prescribed & Telmisartan \\
\hline $\mathbf{1 0}$ & Most commonly two drug therapy prescribed & Telmisartan+ hydrochlorothiazide \\
\hline $\mathbf{1 1}$ & Most commonly three drug therapy prescribed & Telmisartan + amlodipine + hydrochlorothiazide \\
\hline $\mathbf{1 4}$ & Other concomitant medication Prescribed & $\begin{array}{l}\text { Aspirin, atorvastatin, vitamin B complex, thyroxine, } \\
\text { ranitidine, pantaprazole, metformin, glimiperide etc }\end{array}$ \\
\hline
\end{tabular}

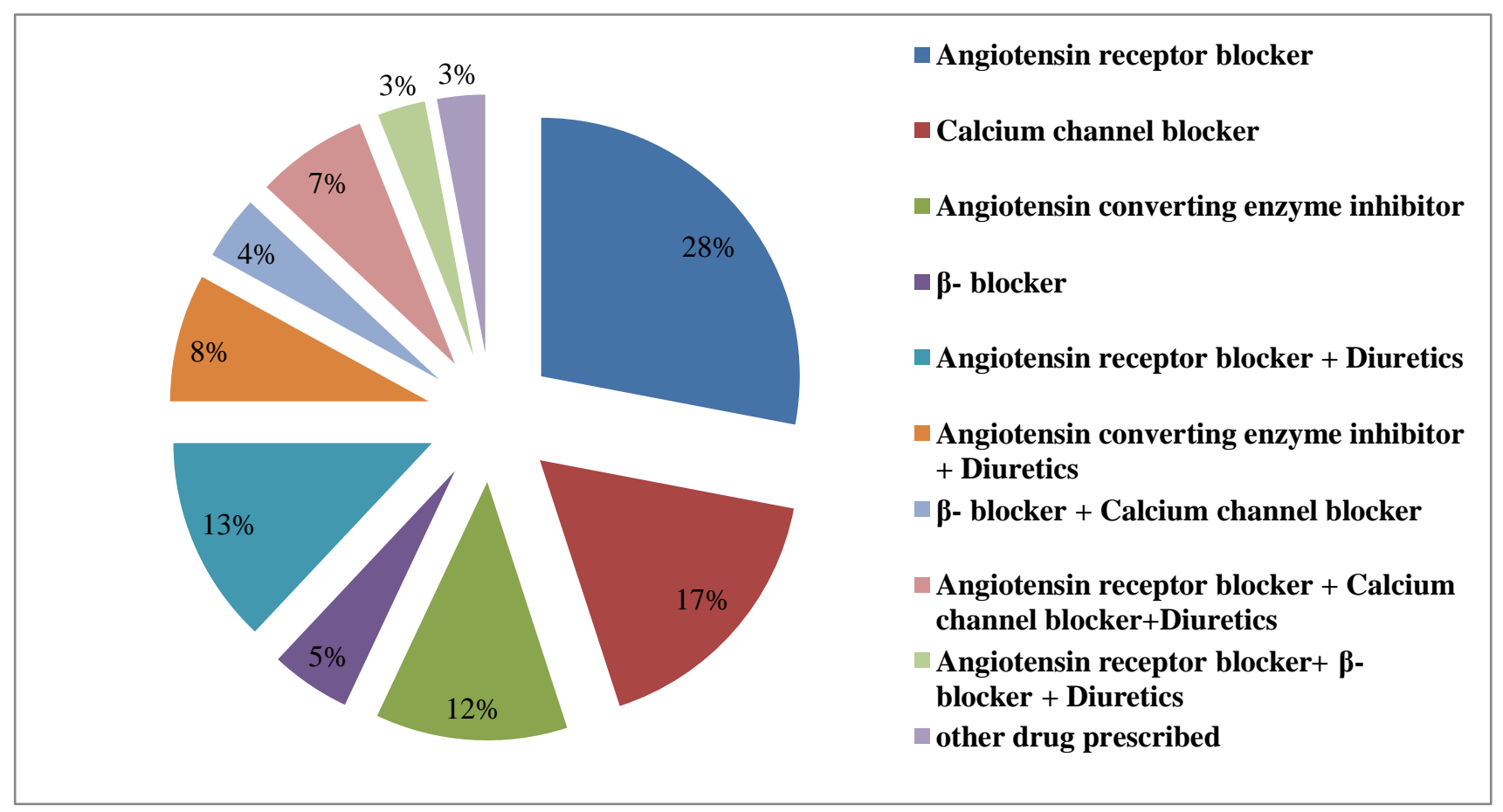

Figure 1: Patterns of antihypertensive drug prescribed.

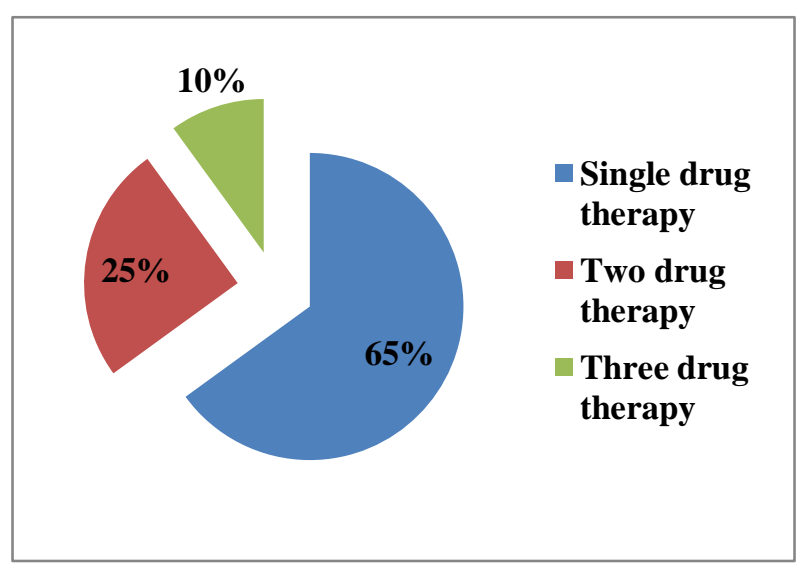

Figure 2: Patterns of drug therapy prescribed in hypertensive patients.

There is various classification among antihypertensive drug prescribed in present study. Among total 246 drug prescribed, Angiotensin receptor blocker was prescribed (ARB) 28\%, calcium channel blocker (CCB) 17\%, Angiotensin converting enzyme inhibitor (ACEI) 12\%,
Beta blocker $5 \%$ followed by ARB + diuretics $13 \%$, ACEI+ diuretics $8 \%$, beta blocker + CCB $4 \%, \mathrm{ARB}+\mathrm{CCB}$ + diuretics $7 \%, \mathrm{ARB}+$ beta blocker + diuretics $3 \%$ and $3 \%$ other drug prescribed (Figure 1).

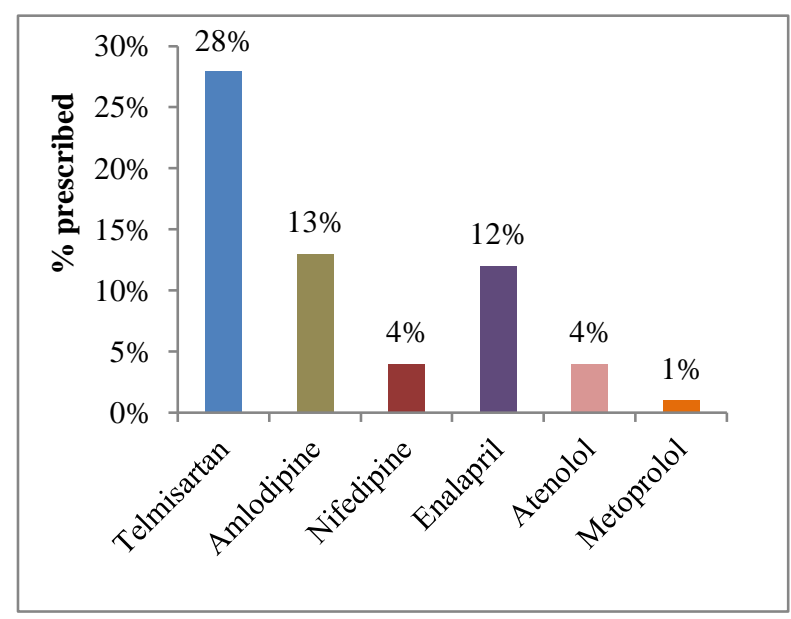

Figure 3: Pattern of single antihypertensive drugs prescribed in patients 
Among total 246 drug prescribed, 65\% single drug prescribed, $25 \%$ two drug prescribed and $10 \%$ three drug prescribed among hypertension patients. Among single antihypertensive drug prescribed, the maximum telmisartan $(28 \%)$ was prescribed followed by amlodipine (13\%), enalapril (12\%), nifedipine (4\%), atenolol (4\%) and metoprolol (1\%) respectively. Among two antihypertensive drug prescribed, $14 \%$ was telmisartan + hydrochlorothiazide, $8 \%$ enalapril + hydrochlorothiazide and $3 \%$ amlodipine + atenolol was prescribed in present study. Among three antihypertensive drug prescribed, $8 \%$ was telmisartan + amlodipine+ hydrochlorothiazide and $3 \%$ was telmisartan + atenolol+ hydrochlorothiazide prescribed. There was no Fixed dose combination prescribed and no injectable prescribed in present study. (Figure 2-5).

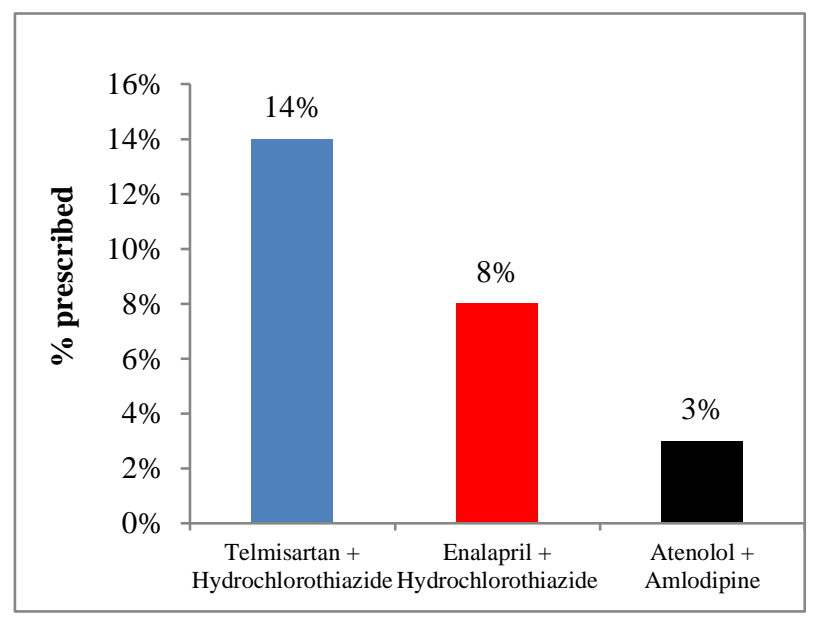

Figure 4: Patterns of two antihypertensive drugs prescribed in patients.

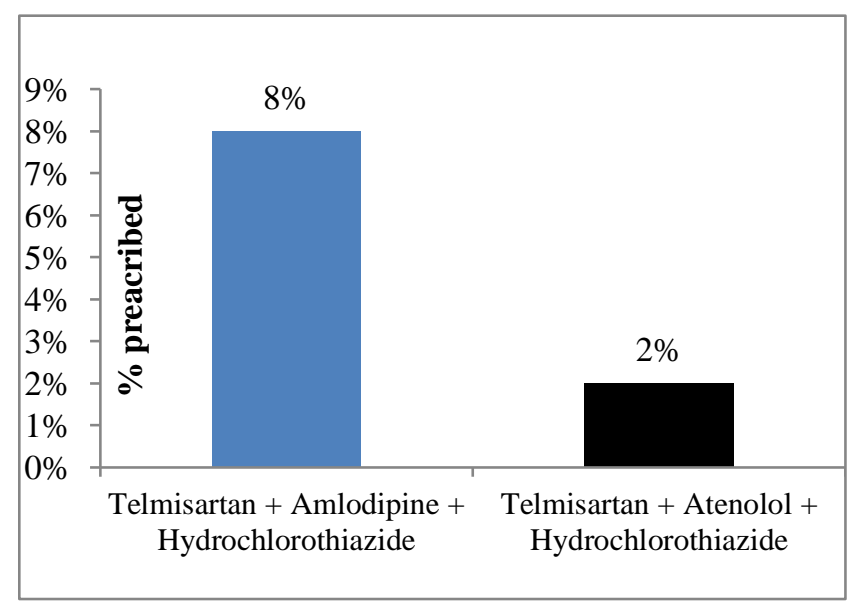

Figure 5: Patterns of three antihypertensive drugs prescribed in patients.

\section{DISCUSSION}

The present study evaluates the prescribing patterns of drugs used in hypertension patients. We analyzed the 100 prescription of the hypertensive patients. We found that the hypertension is more prevalent in male as compare to female. The present study showed $62 \%$ male patients and $38 \%$ female patients. The maximum patients $(42 \%)$ were age group between 61 to 80 years, this age group is more vulnerable to cardiovascular complication. The similar study conducted by Bhavika et al and Gupta et al also concluded the male patients were more as compare with female patients. They both also concluded that the, maximum patients were belonging more than 50 years of age group. The present study showed similar finding as compare with previous studies. ${ }^{7,8}$

The total 246 drugs were prescribed in 100 prescription of hypertensive patients in present study. Average drug per prescription was 2.46. Among 246 drugs, 97\% were antihypertensive drug Prescribed and $3 \%$ were other concomitant drugs prescribed like aspirin, atorvastatin, vitamin B complex, thyroxine, ranitidine, pantaprazole, metformin, glimiperide etc. The most common antihypertensive drug prescribed in present study was telmisartan. Most commonly two antihypertensive drug prescribed was telmisartan + hydrochlorothiazide. Most commonly three antihypertensive drug prescribed was telmisartan + amlodipine + hydrochlorothiazide. The previous study conducted by Bhavika et al, Gupta et al, Sharminder et al and Rajasekhar et al have not calculated average drug per prescription in their study, however, the sample size was more in their study as compare to present study. ${ }^{7-10}$ Bhavika et al reported, amlodipine was maximum drug prescribed as single drug therapy. ${ }^{8}$ The common two drug prescribed was amlodipine + enalapril. The atenolol + enalapril + nifedipine was prescribed as three drug therapy in their study. Which is differ from from present study. The another study by Sharminder et al and Rajasekhar et al, the most common drug as single drug therapy prescribed were angiotensin converting enzyme inhibitor and Calcium channel blocker respectively. ${ }^{9,10}$

There is various classification of antihypertensive drug prescribed in present study. Angiotensin receptor blocker was prescribed (ARB) 28\%, calcium channel blocker (CCB) 17\%, angiotensin converting enzyme inhibitor (ACEI) $12 \%$, beta blocker $5 \%$ followed by ARB + Diuretics $13 \%$, ACEI+ diuretics $8 \%$, beta blocker + CCB $4 \%, \mathrm{ARB}+\mathrm{CCB}+$ diuretics $7 \%, \mathrm{ARB}+$ beta blocker + diuretics $3 \%$ and $3 \%$ other drug prescribed. $65 \%$ single drug prescribed, $25 \%$ two drug prescribed and $10 \%$ three drug prescribed among hypertension patients. Among single antihypertensive drug prescribed, the maximum telmisartan $(28 \%)$ was prescribed followed by amlodipine $(13 \%)$, enalapril (12\%), nifedipine (4\%), atenolol (4\%) and metoprolol (1\%) respectively. Among two antihypertensive drug prescribed, $14 \%$ was Telmisartan + hydrochlorothiazide, $8 \%$ enalapril + hydrochlorothiazide and $3 \%$ amlodipine + atenolol was prescribed in present study. Among three antihypertensive drug prescribed, $8 \%$ was telmisartan + amlodipine + hydrochlorothiazide and $3 \%$ was telmisartan + atenolol+ hydrochlorothiazide prescribed. The previous study showed maximum single drug prescribed from CCB as per Bhavika et and Gupta et 
al and ACE inhibitor as per sharminder et al. ${ }^{9}$ the present study showed maximum drug prescribed from ARB group as antihypertensive drug. The previous study by Bhavika et al showed $64.1 \%$ drug prescribed as single drug therapy, $31.8 \%$ as two drug therapy and $3.9 \%$ as three drug therapy which is almost similar pattern reported by our study. ${ }^{8}$ The rajashekhar et al also showed similar pattern as they reported monotheray $50.76 \%$, dual therapy $42.13 \%$ and triple therapy 6.09 but they also reported four drug therapy also prescribed in their study, which is not prescribed in our study. ${ }^{10}$ The study Gupta et al showed maximum drug prescribed as two drug therapy as $41.9 \%$ and single drug therapy $28.7 \%$ which different pattern from present study. The previous study by Bhavika et al reported the maximum amlodipine + enalapril prescribed as two drug therapy followed by atenolol + enalapril + nifedipine as three drug therapy. The Gupta et al reported the common two drug therapy prescribed was ARB + diuretics followed by ARB + ACEI + diuretics as three drug prescribed. ${ }^{7}$ The rajasekhar et al reported $\mathrm{ARB}+\mathrm{CCB}$ as two drug therapy and $\mathrm{CCB}+\mathrm{ACEI}+\mathrm{CCB}$ as three drug therapy. The present study showed very good pattern to prescribe antihypertensive drug in patients. ${ }^{7,8,10}$

\section{Limitations}

The present study does not calculate cost of the prescribed drug and also co-morbidities among hypertensive patient.

\section{CONCLUSION}

The present study concluded the hypertension is more prevalent in male. The most common drug prescribed was ARB as single drug therapy. The most common drug which was used for combination therapy was diuretics.

Funding: No funding sources

Conflict of interest: None declared

Ethical approval: The study was approved by the Institutional Ethics Committee

\section{REFERENCES}

1. Rachana PR, Anuradha HV, MC Shivamurthy. Antihypertensive Prescribing Patterns and Cost
Analysis for Primary Hypertension: A Retrospective Study. J Clinic Diagnost Res. 2014;8(9):19-22.

2. Lim SS, Vos T, Flaxman AD, Danaei G, Shibuya K, Adair- Rohani H, et al. A comparative risk assessment of burden of disease and injury attributable to 67 risk factors and risk factor clusters in 21 regions, 19902010: a systematic analysis for the Global Burden of Disease Study 2010. Lancet. 2013;380:2224-60.

3. Kearney PM, Whelton M, Reynolds K, Muntner P, Whelton PK, He J. Global burden hypertension: analysis of worldwide data. Lancet. 2005;365:217-23.

4. Etuk E, Isezuo SA, Chika A, Akuche J, Ali M. Prescription pattern of antihypertensive drugs in a tertiary health institution in Nigeria. Ann Afric Med. 2008;7(3):128-32

5. Dighore PN, Patil PH, Kuchake VG, Maheshwari OD. Prescription patterns of antihypertensive drugs. Ind $\mathbf{J}$ Hospital Pharm. 2009;46:78-82.

6. Sikidar P, Chakravarty P, Purkayasyha A, Tigga R. A study on prescribing pattern of antihypertensives in adult patients attending in a tertiary care hospital of Assam, India. Int J Basic Clin Pharmacol. 2016;5:9758.

7. Gupta R, Malhotra A, Malhotra P. Study of prescribing pattern of drugs used in the treatment of hypertension in a tertiary care teaching hospital in North India: an observational study. Int J Res Med Sci 2018;6:2380-4.

8. Bhavika D, Prasanna V, Swathi B. Drug utilization study of anti-hypertensive drugs in a tertiary care hospital. Int J Basic Clin Pharmacol 2016;5:1580-5.

9. Kaur S, Gupta S, Kumar D, Lal M, Gilani Z. Prescribing pattern of antihypertensive drugs in a tertiary care hospital in Jammu-A Descriptive study. JK-Practit. 2012;17(4):38-41.

10. Giri rajasekhar D, Guru prasanna D, Chandrakanth P. Prescribing pattern of antihypertensive drugs based on compelling indications with hypertension. Int J Pharm Pharm Sci 2019;8(2):72-5.

Cite this article as: Suman RK, Singh HK, Patil VG. Prescribing patterns of antihypertensive drugs in tertiary care teaching hospital. Int J Basic Clin Pharmacol 2021;10:420-4. 